\title{
Chromatic effects in Hipparcos parallaxes and implications for distance scale
}

\author{
Dimitri Pourbaix $\dagger$ \\ Institute of Astronomy and Astrophysics, Université libre de Bruxelles CP 226, B-1050 \\ Brussels, Belgium email: pourbaix@astro.ulb.ac.be \\ and \\ Department of Astrophysical Sciences, Princeton University, Princeton NJ 08543-1001, USA
}

\begin{abstract}
The change in the measured position due to the chromatic effect in the optical system is usually so small that it can be neglected. However, in the case of the Hipparcos mission, this effect is of the same magnitude as the targeted precision; it is therefore mandatory to correct for it. The different assumptions adopted to build up such corrections in the Hipparcos framework are presented. Some of their limitations and how they can be overcome to improve the results even today are described. The chromaticity mainly affects red stars and has its strongest impact on the astrometry of very red and highly variable stars such as Miras. Nevertheless, the implications on distance scale determination are rather small.
\end{abstract}

\section{Introduction}

The Hipparcos Catalogue (ESA 1997) contains parallaxes of nearly 120000 stars brighter than $V \approx 12.4$, up to roughly $1 \mathrm{kpc}$ away. In order to achieve a very high precision (and hopefully accuracy) in the derived quantities, several corrections were applied to the raw observations, including some to account for the chromaticity effects.

Owing to the pressure of releasing the catalogues, some simplifying assumptions were adopted in the reductions. For instance, a fixed color and, therefore, a constant chromatic correction was assumed for each individual star. Though sound for most stars, this assumption apparently is not correct, for instance, in the case of red long period variable stars such as Miras, LPVs (Mira-like, SR, ...) and may result in biased parallaxes.

In this contribution, we first note that the number of binaries in the Hipparcos catalogue is correlated with the color of the object - the redder the color the higher the likelihood to be a binary. We describe the way the chromaticity was originally corrected for in the Hipparcos framework and show that the connection between the color and binarity most likely is an artifact of the original assumption about the fixed color of stars. We then focus on how those initial results can benefit from ground-based photometric data and how, once combined together, they improve the accuracy of parallaxes, hence, the distance scale for long-period red variable stars.

\section{Duplicity and color}

Wielen $(1996,1997)$ suggested that unresolved binaries with one variable component would exhibit a motion of the photocenter correlated with the overall brightness change. An annex of the Hipparcos Catalogue, namely the DMSA/V, is dedicated to such objects called VIM, for Variability-Induced Movers. For all of them, it was assumed that the

$\dagger$ Research Associate, FNRS, Belgium 


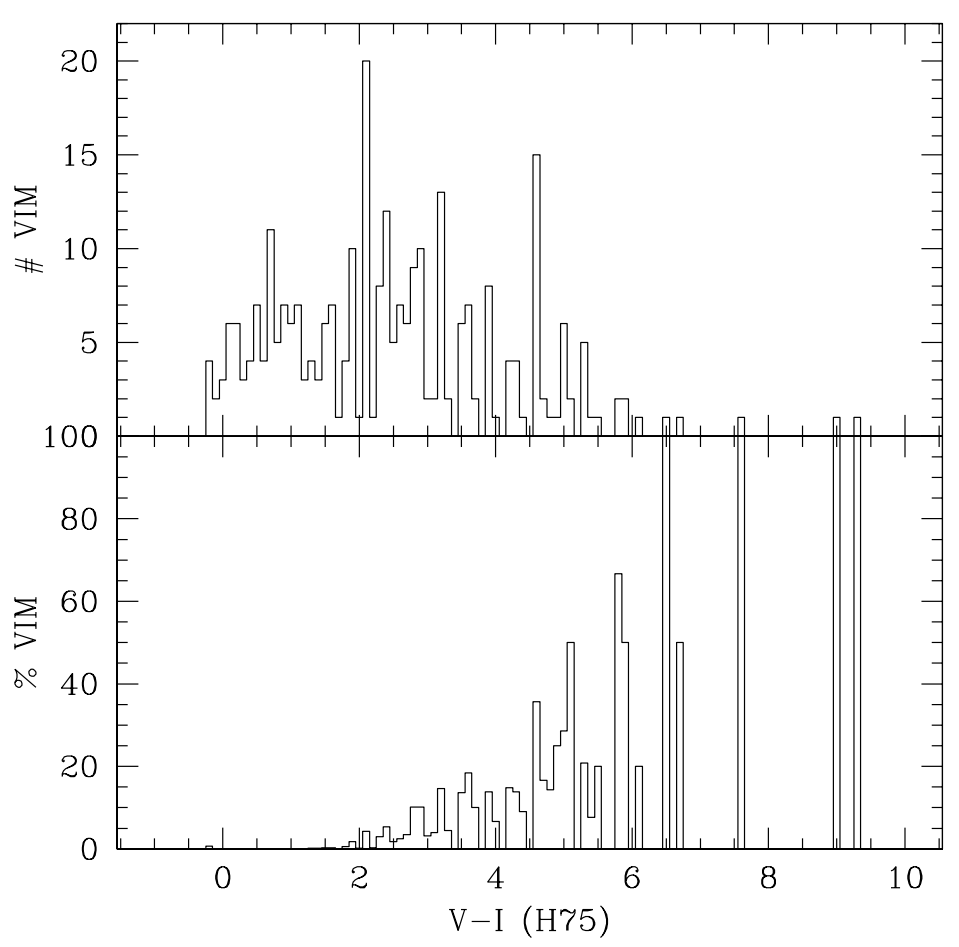

Figure 1. Distribution of the $V-I$ color of VIM. The top panel represents the actual number of VIM whereas the bottom panel gives the distribution of the percentage of VIM among all Hipparcos stars of similar color.

orbital period was long enough for the relative motion of one component with respect to the other to be negligible over the mission lifetime.

The position of the photocenter $(\xi, \eta)$ is thus given by:

$$
\begin{aligned}
& \xi=\alpha_{0}^{*}+\mu_{\alpha^{*}}\left(t-t_{0}\right)+P_{\alpha} \varpi+\left(10^{0.4\left(H p_{t o t}(t)-H p_{r e f}\right)}-1\right) D_{\alpha^{*}}, \\
& \eta=\delta_{0}+\mu_{\delta}\left(t-t_{0}\right)+P_{\delta} \varpi+\left(10^{0.4\left(H p_{\text {tot }}(t)-H p_{\text {ref }}\right)}-1\right) D_{\delta}
\end{aligned}
$$

where $\alpha_{0}^{*}, \delta_{0}, \varpi, \mu_{\alpha^{*}}, \mu_{\delta}$ are the five fundamental astrometric parameters (position, parallax and proper motion), $P_{\alpha}, P_{\delta}$ are the parallactic factors, $H p_{t o t}(t)$ and $H p_{r e f}$ are respectively the total $H p$ magnitude at time $t$ and a reference magnitude. $D_{\alpha^{*}}$ and $D_{\delta}$ are the components of the direction of the secondary with respect to the primary star. A VIM object thus requires a 7 -parameter model where $D_{\alpha^{*}}$ and $D_{\delta}$ are obtained together with $\alpha_{0}^{*}, \delta_{0}, \varpi, \mu_{\alpha^{*}}$ and $\mu_{\delta}$.

Though this model is legitimate and one expects such binaries to be present in a large stellar sample such as the Hipparcos catalogue, the color distribution of those VIMs are rather unexpected, as shown in Fig. 1. The percentage of VIMs is clearly color dependent: the redder the star, the higher the percentage. If VIMs are indeed binaries, that would mean that red stars are more likely to be binaries which no other investigation has shown. A similar correlation between the color and binary percentage was also noticed by Platais et al. (2003) on a larger sample of Hipparcos stars.

Rather than questioning the binary distribution/likelihood over the Hertzsprung-Russell diagram, it might be worth looking for some neglected instrumental effects or biases 
arising from the data reduction. For instance, what role does the color of an object play in the reduction, what were the assumptions?

\section{Hipparcos chromaticity correction}

Like any optical device with refracting elements, the Hipparcos satellite was subject to chromatic effects, i.e. light rays at different wavelengths follow different paths and therefore hit slightly different parts of the detector. While such an offset is so small that it can be safely ignored in most applications, it had to be accounted for and corrected in order to reach the very high precision aimed at by Hipparcos.

One can assume that the offset in position (for Hipparcos - along the scanning direction) is proportional to the offset in color:

$$
\text { Offset }=p_{0}\left(C_{*}-C_{\text {Ref }}\right)
$$

where $C_{*}$ denotes the color of the object and $C_{R e f}$ is the reference color for which zero chromatic displacement is adopted. In the case of Hipparcos, a term was added to account for aging of the instrument, i.e. a gradual evolution of detector's response function over the lifetime of the mission:

$$
\text { Offset }=p_{0}\left(C_{*}-C_{\text {Ref }}\right)+p_{1}\left(C_{*}-C_{\text {Ref }}\right)(t-1991.25) .
$$

Since Hipparcos did not carry an on-board multi-band photometer, the ground-based colors were adopted. Initially built upon $B-V$ colors, the $V-I_{C}$ color index was finally adopted as a more reliable color indicator for the Hipparcos sample. While both data reduction consortia (FAST and NDAC) used $V-I_{C}$, they had their own chromaticity correction expressions. For instance, FAST had an additional quadratic term in Eq. 3.2 whereas NDAC calibrated the correction a function of effective wavelength which was used in lieu of the $V-I$ color index.

However, the $V-I_{C}$ color for all $\sim 120000$ stars was not more readily available than the $B-V$ color. The adopted values were either directly measured from ground (2929 stars) or derived from other colors, e.g. $B-V$. Nineteen different methods for such a derivation were used depending on the spectral type and luminosity class of stars. Thus, $97 \%$ of the chromaticity corrections are based on the empirically estimated $V-I_{C}$ colors. And yet, the worst is still to come.

As illustrated in Fig. 2, a change in magnitude often yields a change in color. Although the amplitude of the latter is not as large as of the former, it can still cause a shift of several milli-arc seconds (mas) across the detector. If this variable shift is unaccounted for, the position of the photocenter is apparently correlated with the brightness. This is a typical case of VIM but without a real secondary. It is thus likely that a fraction of the VIMs, especially the red ones, are just an artifact of the reduction pipeline and should rather be called Color-Induced Movers. In order to assess this alternative explanation, one needs to apply a better chromaticity correction due to the change in color and then decide whether the VIM model is still worth adopting. While removing the original chromaticity correction and applying a new one based on a variable color are rather straightforward (at least for the second part), deriving an instantaneous color is much more difficult as we see in the following section.

\section{Deriving $V-I_{C}$ for red Hipparcos stars}

How do we obtain $V-I_{C}$ color for each individual Hipparcos observation despite the fact that no on-board instrument measured that color and no parallel ground-based 


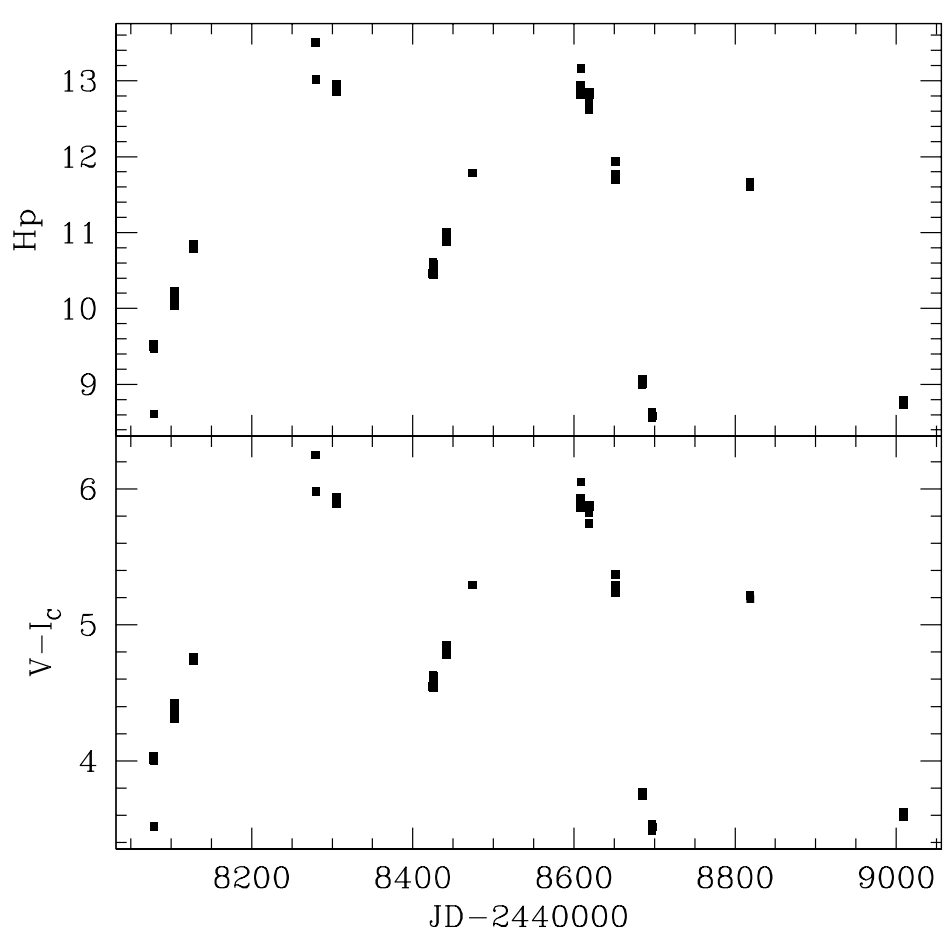

Figure 2. Variability in magnitude and color.

observing campaign was set up for that purpose? We here outline how the instantaneous Cousins $V-I$ were "restored" from a combination of the ground-based and Hipparcos/ Tycho observations (Platais et al. 2003).

First, we collected all the existing $V-I$ for a large sample of $\mathrm{M}, \mathrm{S}$, and $\mathrm{C}$ spectral type stars. Some of these $V-I$ were already published while other were measured for this purpose. Whenever necessary, those colors were changed into the natural Cousins system using color-color transformation listed in Volume 1 of the Hipparcos catalogue. The result was a set of $567 V-I_{C}$ color indices. Although this sample already makes it possible to assess the accuracy of the adopted Hipparcos $V-I_{C}$, it does not address the variability of this color index over the mission lifetime.

It was noticed that, for reasonably high fluxes a new instantaneous color index for red stars, $H p-V_{T 2}$, is strongly correlated with $H p$, and can be well-modeled for each such star with a straight line.

$$
H p=\frac{b_{0}+V_{T 2}}{1-b_{1}} .
$$

Those lines thus express $H p$ in terms of $V_{T 2}$. Volume 1 of the Hipparcos catalogue also lists transformations from $V-I_{C}$ to $V_{T}$ (measured as $V_{T 2}$ ). Combining all these results, Platais et al. (2003) therefore established a color-color transformation from $V-I_{C}$ to $H p-V_{T 2}$. It is represented by two fourth-order polynomials (Fig. 3) depending on the spectral type of the stars.

Such a tight relationship now makes it possible to derive the $V-I_{C}$ color for any epoch. Using this relationship for a given red star, a set of Hipparcos and Tycho photometric 


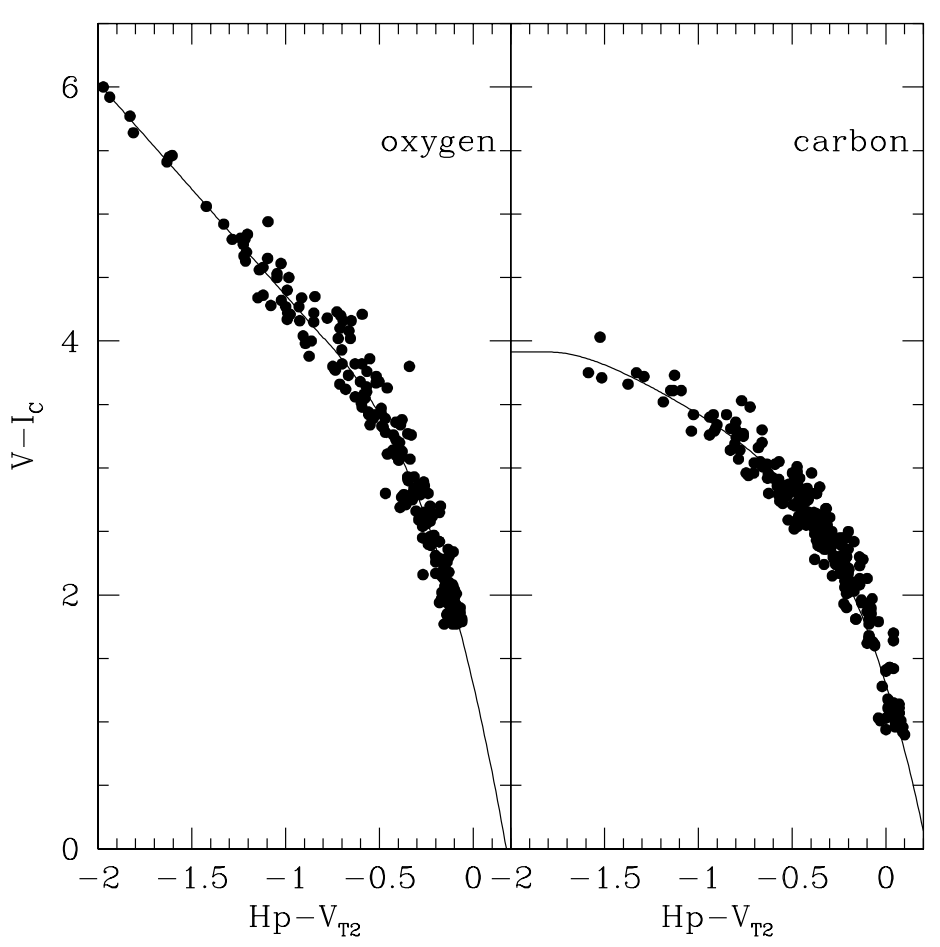

Figure 3. Color-color diagram of oxygen and carbon long period variable stars.

observations themselves allow us to predict what was the corresponding instantaneous $V-I_{C}$ color index at each epoch of Intermediate Astrometric Data.

\section{Revised model and parallaxes}

Once the observations are properly corrected for chromaticity thanks to the significantly improved colors, one can evaluate how the number of VIMs changes and, if the adopted model is different, how this affects the parallax. According to what is stated in the previous section, most VIMs are actually color-induced movers and, therefore, should disappear when correct colors are adopted.

The evolution of the distribution of most VIMs is given in Fig. 4. The first thing to notice is that the number of VIMs falls by $20 \%$ if, instead of using FAST astrometric data (as originally done), one takes advantage of the NDAC part as well (upper left corner). Only 188 VIMs are carbon or oxygen LPVs for which epoch $V-I_{C}$ were derived. Using FAST and NDAC data, 150 VIMs survive (upper right corner). So, even without changing anything to the chromaticity correction, just by adding the NDAC data, $21 \%$ of the VIM get already discarded. The lower panels of Fig. 4 present the results when epoch $V-I_{C}$ are used. The number of VIMs is given for two values of the statistical confidence (10\% which correspond to the original one and $0.27 \%$ ). The left panel uses the original $V-I$ on its x-axis whereas the right panel uses the median of the epoch $V-I_{C}$ instead. Clearly, the number of VIMs drops (by at least 47\%) and the red tail of the distribution simply disappears.

Among the original VIMs, using epoch $V-I_{C}$ often leads to the replacement of the VIM model by the single star model, thus potentially affecting the parallax, especially 


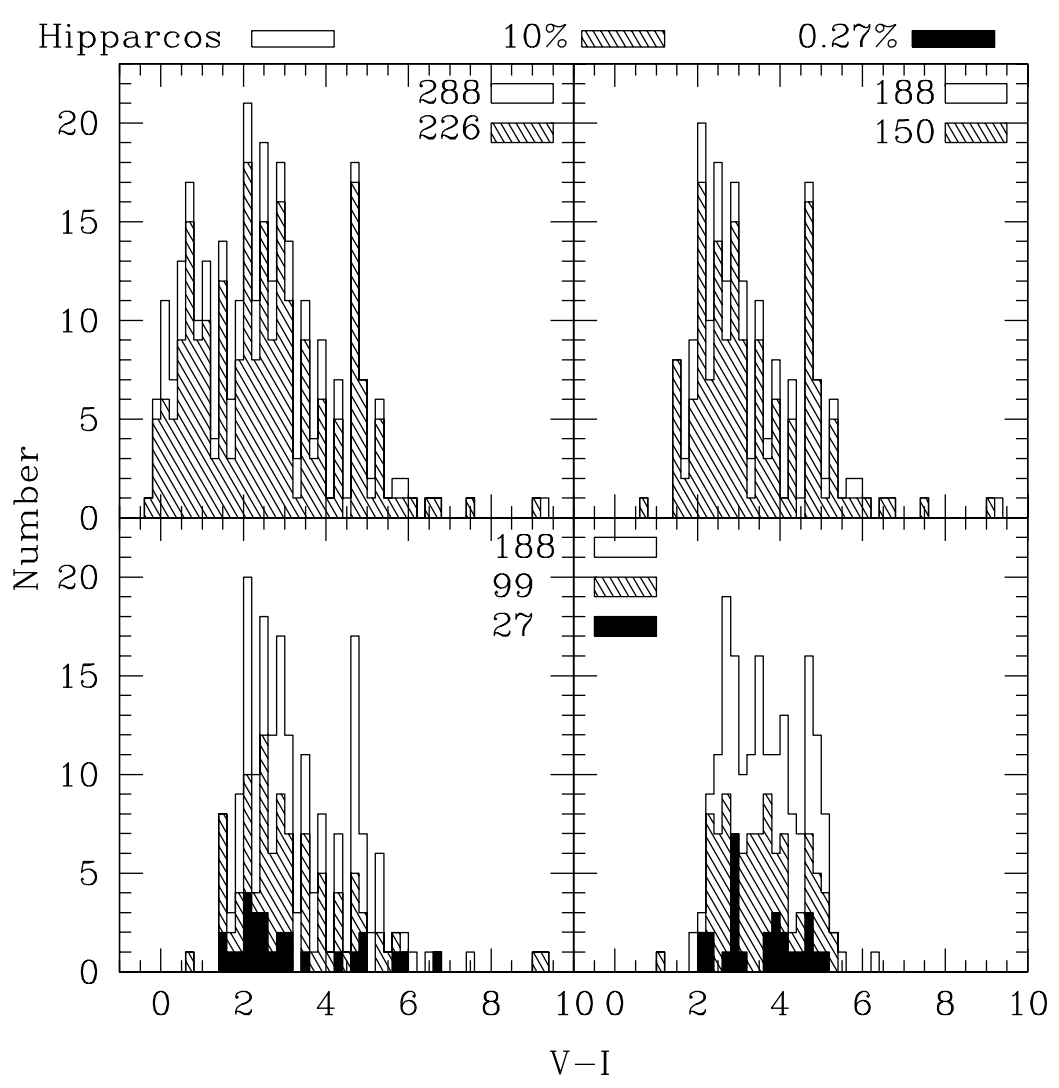

Figure 4. Evolution of the distribution of VIMs in terms of $V-I_{C}$

for those variable stars with periods close to one year. Even for the objects originally processed as single stars, the revised chromaticity correction potentially changes the astrometric solution and its uncertainty.

Once compared with independent determinations of the distance of LPVs (e.g., Mennessier \& Luri 2001), some of the newly derived parallaxes are in much better agreement than were the original ones. For example, the parallax of V1943 Cyg changes from $1.82 \pm 1.82$ mas to $5.02 \pm 0.97$ mas - versus 4.7 mas (Mennessier \& Luri 2001; Neugebauer \& Leighton 1969); R Hya from $1.62 \pm 2.43$ mas to $8.44 \pm 1.00$ mas - versus 7.14 mas (Whitelock et al. 2000).

One way of extending the Hipparcos results beyond $1 \mathrm{kpc}$ consists in using its parallaxes to derive the Period-Luminosity relation of Mira-like variables. Adopting the slope obtained in the LMC (Feast et al. 1989), van Leeuwen et al. (1997) derived the zero-point of the relation based upon the Hipparcos parallaxes of 16 pre-selected Mira variables and obtained:

$$
M_{K}=-3.47 \log P(\text { days })+0.94 \pm 0.18,
$$

where $M_{K}$ denotes the absolute $K$ magnitude and $P$ the period of variability. From a sample of 255 Mira-like variables and still adopting the slope from Feast et al. (1989), Whitelock \& Feast (2000) derived:

$$
M_{K}=-3.47 \log P(\text { days })+0.84 \pm 0.14
$$



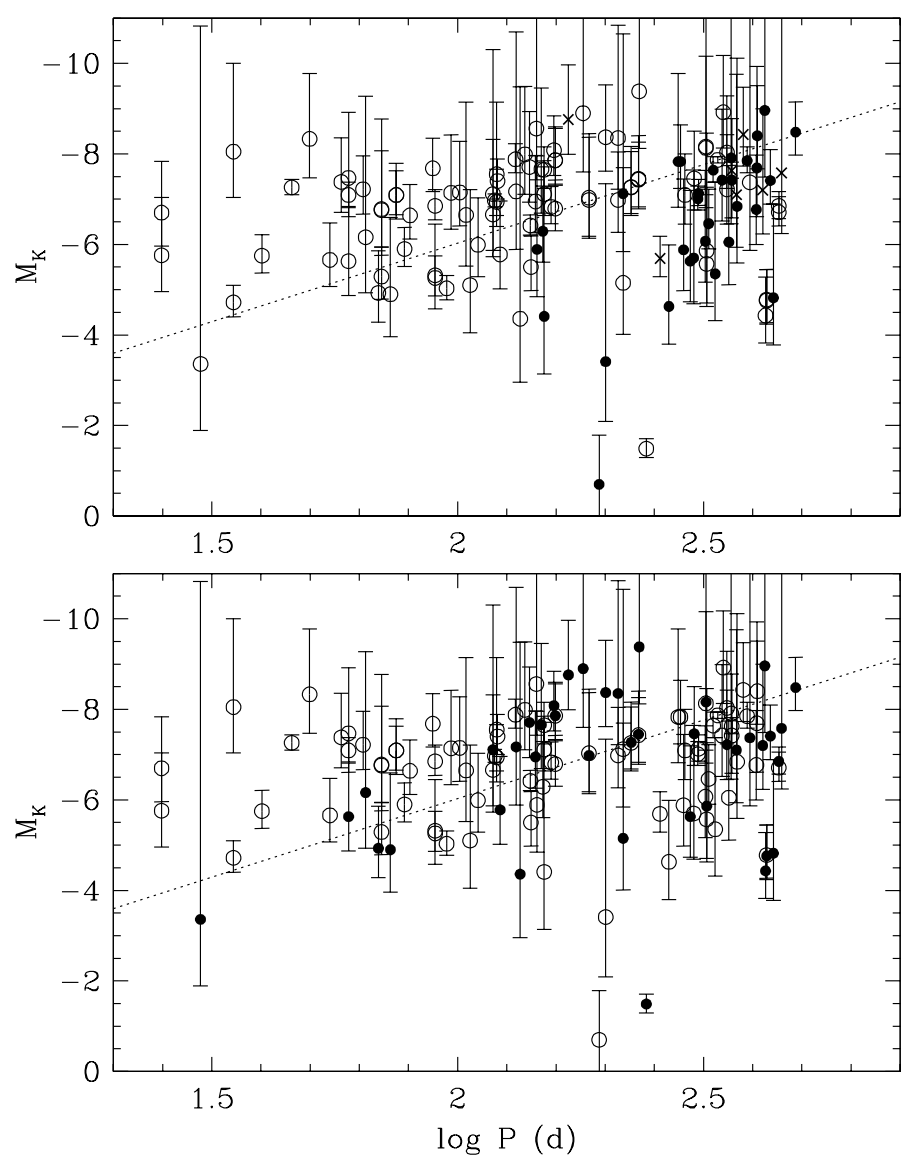

Figure 5. Distribution of LPVs in the $M_{K}-\log P$ plane. Upper panel: stars are sorted by variable type - filled circles $=$ Miras; open circles $=\mathrm{SR} / \mathrm{SRb}$; crosses $=\mathrm{SRa}$. Lower panel: stars are sorted by chemical type - filled symbols = carbon stars; open symbols = oxygen stars; the dashed line is the P-L relationship of Eq. (5.2).

All previous derivations assume the empirical LMC slope, not really knowing whether it also applies to Galactic Miras. In order to establish the soundness of that approach, Knapp et al. (2003) derived both the slope and the zero-point from the Hipparcos data revised with the new chromaticity correction. Fitting

$$
\varpi=P^{\frac{a}{5}} 10^{\frac{b}{5}} 10^{\frac{10-K+A_{K}}{5}}
$$

instead of

$$
M_{K}=a^{\prime} \log P(\text { days })+b^{\prime}
$$

because of the Gaussian error on $\varpi$ only, they obtain

$$
M_{K}=-3.39( \pm 0.47) \log P(\text { days })+0.95( \pm 3.01) .
$$

$A_{K}$ denotes the interstellar absorption in the $K$ band. Although the parallaxes are accurate enough to yield a relation consistent with the previous ones, the uncertainty on the parallaxes is still way too large to precisely derive both the slope and the zero-point. 


\section{Conclusions}

We have shown that a simplistic assumption about fixed colors of many red variable stars over the Hipparcos mission yielded some questionable parallaxes together with a strong correlation between the color and binary percentage. A nice feature of the Hipparcos catalogue is the availability of the low level data which make it possible to improve the original data processing upon the availability of new data.

Although ESA had not foreseen that the chromaticity correction could be revised as well, Platais et al. (2003) nevertheless succeeded in taking advantage of ground-based $V-I_{C}$ colors to improve the correction of the chromatic effects. Thanks to these improved colors, the number of stars seemingly best described as VIM vanished quite substantially. The (default) single star model not only fits the data at a satisfactory level but, in some cases, it also improves the parallax accuracy.

Regardless of which model presently fits the observations, the revised parallaxes can be used to derive the parameters of the period-luminosity relationship for long-period variables. The LMC slope is confirmed (unlike other investigations where that slope is simply assumed) but those parallaxes are neither accurate enough nor precise enough to reliably adjust both the slope and the zero-point.

\section{Acknowledgements}

This research was supported in part by ESA/PRODEX C15152/01/NL/SFe(IC) and by NASA via grant NAG5-11094.

\section{References}

ESA 1997, The Hipparcos and Tycho Catalogues. ESA SP-1200

Fabricius C., Makarov V. V., 2000, A\&A, 356, 141

Feast M. W., Glass I. S., Whitelock P. A., Catchpole R. M., 1989, MNRAS, 241, 375

Høg E., Fabricius C., Makarov V. V., Urban S., Corbin T., Wycoff G., Bastian U., Schwekendiek P., Wicenec A., 2000, A\&A, 355, L27

Knapp G. R., Pourbaix D., Platais I., Jorissen A., 2003, A\&A, 403, 993

Mennessier M. O., Luri X., 2001, A\&A, 380, 198

Neugebauer G., Leighton R. B., 1969, Two-Micro Sky Survey Catalogue. NASA SP-3047

Platais I., Pourbaix D., Jorissen A., Makarov V. V., Berdnikov L. N., Samus N. N., Lloyd Evans T., Lebzelter T., Sperauskas J., 2003, A\&A, 397, 997

Pourbaix D., Platais I., Detournay S., Jorissen A., Knapp G., Makarov V. V., 2003, A\&A, 399, 1167

van Leeuwen F., Feast M. W., Whitelock P. A., Yudin B., 1997, MNRAS, 287, 955

Whitelock P., Feast M., 2000, MNRAS, 319, 759

Whitelock P., Marang F., Feast M., 2000, MNRAS, 319, 728

Wielen R., 1996, A\&A, 314, 679

Wielen R., 1997, A\&A, 325, 367

\section{Discussion}

FloOR VAN LEeUWEN: I would like to make a few clarifications. In the science team for Hipparcos, when we were approaching the publication of the data it was decided that it was better to keep $V-I$ constant than to try to put in a variable $V-I$ which was uncertain. So we kept everything constant with the idea that it could be corrected more easily from that value than from a variable input. We didn't have the time to derive the epoch $V-I$ photometry that you have been deriving. So that was the best we could do. This is not a question of a mistake. This is a question of a purposeful best comprise. That's why we provide both the $V-I$ as used in the reduction, and what was 
afterwards thought of as the best $V-I$; you find both values there. Secondly, the work that you've been doing, it is worth noticing, is all incorporated in the new reduction [see van Leeuwen, these proceedings]. So the $V-I$ variability is all already incorporated in the new reduction, and doing a very good job. I can see that all the calibrations that concerned the colour are a lot better than they have been before. Because $V-I$ not only works through what you have been looking at, but it also works at a much deeper level where the data are not available for correction.

Mikhail Marov: You introduced the covariance matrix and it's very powerful for the data processing. How well did the data fit along the main diagonal and how large is the scatter? Are the scattered points meaningful, or just noise?

Dimitri Pourbaix: I only mentioned one covariance matrix, the one given in the Hipparcos Catalogue, so that's something I can't change. The only thing I can change in that relation is the $p$, the $\Delta p$. So, for instance, if I take the right ascension, I know the value published for the right ascension. I will try another one; the difference between the two will give me the $\Delta p_{1}$. So that's the only place where I have room for change. I can't change the $V$ there. The covariance matrix is given from the reduction; so it's just the weight of all the observations. I have no room for improvement there.

Mikhail Marov: So, it has nothing to do with the multi-band photometry?

Dimitri Pourbaix: No.

FRITZ BENEDICT: You threw out about $90 \%$ of those VIMs as possible binaries, and you did mention that the navy was going after them. Did their statistics agree with yours? Did they get about a $10 \%$ hit rate while looking at them?

Dimitri Pourbaix: The problem is, in their case, they confirm a binary if they see two components. If they don't see two components, they can't say anything. They can just say, "no detection"; that's all. What we did was to reduce the effort in looking for new binaries.

RickY SmaRT: You got a calibration for $H-V$ for Miras. Did you apply it to everything?

Dimitri Pourbaix: No. Only to Miras.

RICKY SMART: So all your VIMs are Miras?

Dimitri Pourbaix: Most of them, yes. For the VIMs we look only for Miras, and that's indeed why you expect most of them, since you need a big change in brightness in order to see a displacement.

RickY SmarT: Why did you use the $H-V$, which are quite close bands, instead of the $B-V$ from Tycho?

Dimitri Pourbaix: That's where we got the maximum flux.

Floor Ven LeEuwen: The main benefit here is that the $H$-band is actually extending quite nicely towards the red. The difference between the $V$ and the $H$ band is the red wing. 